\title{
Prevalence of MDR1 C3435T and CYP2B6 G516T polymorphisms among HIV-1 infected South African patients
}

\author{
Tracy Madimabi Masebe ${ }^{\mathrm{a}}$, Pascal Obong Bessong ${ }^{\mathrm{a}, *}$, Julius Nwobegahay $^{\mathrm{a}}$, Roland Ndip Ndip ${ }^{\mathrm{b}, \mathrm{c}}$ and \\ Debra Meyer ${ }^{\mathrm{d}}$ \\ ${ }^{a}$ AIDS Virus Research Laboratory, Department of Microbiology, University of Venda, Thohoyandou, South Africa \\ ${ }^{\mathrm{b}}$ Department of Biochemistry and Microbiology, University of Fort Hare, Alice, South Africa \\ ${ }^{\mathrm{c}}$ Department of Life Sciences, University of Buea, Buea, Cameroon \\ ${ }^{\mathrm{d}}$ Department of Biochemistry, University of Pretoria, Pretoria, South Africa
}

\begin{abstract}
Data on genetic polymorphisms associated with response to anti-HIV drugs has accumulated over the years. Information on how polymorphisms influence drug metabolism and transport to target sites is important in guiding dosage or selection of appropriate alternative therapies. This study determined the frequency of MDR1 C3435T and CYP2B6 G516T polymorphisms associated with the transport and metabolism of efavirenz and nevirapine, in a population of South African HIV infected patients. In addition, association of polymorphisms with immunologic and virologic factors was investigated. A 207bp of MDR1 exon 26 and a 161bp of CYP2B6 exon 4 were obtained from patients by polymerase chain reaction. Analysis of population-based sequences of MDR1 revealed a frequency of $89 \%$ and $11 \%$ of $\mathrm{C}$ and T alleles respectively $\left(n=197 ; \mathrm{X}^{2}=0.974 ; p=0.324\right)$. Restriction fragment length polymorphism (RFLP) analysis of the CYP2B6 gene revealed a prevalence of 9.5\% of GG, 78.4\% of GT and $12.1 \%$ of TT genotype $\left(n=199 ; \mathrm{X}^{2}=65.204 ; p=0.00\right)$. There was no significant difference between immune recovery and decline in viral load $(n=53)$, with genotype after repeated calculations of analysis of variance (ANOVA).
\end{abstract}

Keywords: HIV, MDR1, CYP2B6, polymorphisms, South Africa

\section{Background}

Acquired immunodeficiency syndrome (AIDS) is one of the greatest infectious disease challenges in human history. In order to curb this scourge, highly active antiretroviral therapy (HAART) is used, and has greatly improved AIDS prognosis. Long-term efficacy and toxicity are major issues of concern when selecting a treatment regimen for AIDS. In addition, genetic variability of individuals may lead to differences in pharmacodynamics and pharmacokinetics of drugs, thus complicating therapy at the individual level. These differences can affect efficacy thereby contributing to

*Corresponding author: Pascal Obong Bessong, AIDS Virus Research Laboratory, Department of Microbiology, University of Venda, PMB X5050 Thohoyandou 0950, South Africa. Tel.: +27 15962 8301; Fax: +27 15962 4749; E-mail: bessong@univen.ac.za. therapeutic failure $[1,2]$. Other factors such as lack of compliance and drug toxicity are also implicated as obstacles in treatment selection [2].

The major determinants of drug bioavailability include drug transporters and metabolisers such as Pglycoprotein (P-gp) and the cytochrome P450 system (CYP450). However, several polymorphisms that alter the protein product involved in metabolism, transport, absorption and excretion of many drugs have been described [1]. The combined activity of CYP450 and Pgp may be responsible for the low and variable bioavailability of a range of drugs [3]. Oral absorption and tissue penetration of efavirenz (EFV) and nevirapine (NVP), non-nucleoside reverse transcriptase inhibitors (NNRTI); and nelfinavir, ritonavir and saquinavir, protease inhibitors (PIs) [4] are influenced by the P-gp transporter encoded by MDR1 gene [2].

The current South African treatment guidelines recommend the use of tenofovir (TDF), lamivudine (3TC) 
and EFV/NVP as first line therapy; and zidovudine (AZT), 3TC, and lopinavir/ritonavir as the second line regimen [5]. EFV and NVP are principally metabolized by the CYP2B6 system; EFV is metabolized to hydroxylated metabolites with subsequent glucuronidation and the drug has a long plasma half-life [6,7].

CYP2B6 is an isoenzyme found on exon 4 of chromosome 19 and is characterized by wide inter-individual and inter-ethnic variation in expression and activity in the human liver in vitro [8]. The presence of a $\mathrm{G}>\mathrm{T}$ single nucleotide polymorphism (SNP) at position 516 of CYP2B6 gene results in a Gln-His (GlutamineHistidine) amino acid change associated with higher plasma EFV concentrations leading to increased drugrelated side effects [9-13]. This change is associated with a significant reduction in CYP2B6 catalytic activity [14]. Whetham and colleagues [15] reported higher EFV concentrations associated with higher, but usually therapeutic plasma EFV level in South African men with the TT genotype.

Inter-individual variability of P-gp expression may affect the rate of immune recovery after antiretroviral therapy intake [16]. It is an ATP-dependant efflux transporter of multiple hydrophobic low molecular weight drugs, metabolites and other compounds. Its expression in CD4 $\mathrm{T}$ cells and in the blood brain area can limit the entry of protease inhibitors [17].

The C3435T change at a wobble position in exon 26 on chromosome 7 of the human genome has pharmacological consequences, and has been reported in a number of African populations and other ethnic groups in different populations $[4,18]$. The frequency of the C3435T mutation is significantly influenced by ethnicity with marked differences in genotypes seen between different populations. Several studies have reported a high prevalence of the CC genotype in different African populations, and this prevalence implies overexpression of P-gp [18,19]. In individuals with CC genotype, access of HIV protease inhibitors to major cellular targets known to express P-gp is restricted and this could have serious implications in the use of protease inhibitors [4,20]. Patients with the T homozygous genotype have been shown to have low expression of P-gp. The C3435T SNP is also correlated with P-gp expression and function on lymphocytes but not on placenta [21]. Several studies have reported significantly greater CD4 cell count in patients with the MDR1 3435TT genotype and these patients tend towards less pronounced viral infection than those patients with the CT or CC genotype [22,23].

Characterization of MDR1 and CYP2B6 enzymes and utilization of pharmacogenomic testing for iden- tification of different alleles in patients may provide a useful tool for therapy optimization with drugs that are substrates of P-gp and those that are metabolised through the CYP2B6 pathway [24]. Chelule and colleagues [18] reported an $85.9 \%$ prevalence of MDR1 CC genotype among Africans, $41.7 \%$ in Indians and $35.7 \%$ in whites in KwaZulu Natal, South Africa. However, data on the role of CYP2B6 G516T and MDR1 C3435T in predicting therapy outcome in HIV-infected South African patients is scarce, and therefore compiling information on polymorphisms in this population may be essential in elucidating inter-individual variability in drug responses. Thus, this study was carried out to determine the frequency of major known variant alleles and genotype distribution of MDR1 C3435T and CYP2B6 G516T polymorphisms, as well as correlating observed polymorphisms with the immunologic and virologic responses in an HIV-1 infected South African population.

\section{Methods}

\subsection{Study population}

Study volunteers were HIV infected individuals who were prospectively recruited from January 2008 to March 2009 from two HIV/AIDS treatment centres in Limpopo province, South Africa: Bela-Bela AIDS Wellness Clinic (Waterberg District) and the Phela O Phedishe (POP) Clinic in Mankweng Hospital (Capricorn District) situated in northern South Africa. According to the South African 2001 census data, the racial composition of Limpopo Province was $96.9 \%$ African/Black, $0.19 \%$ coloured (mixed race), $0.17 \%$ Indian/Asian, and $2.6 \%$ white (Caucasian). This is comparable with KwaZulu Natal Province which had 85\% African/black, $1.4 \%$ coloured (mixed race), $8.3 \%$ Indian/Asian, and $5.0 \%$ white (Caucasian). Western Cape Province had 26.0\% African/black, 53.0\% coloured (mixed race), $0.99 \%$ Indian/Asian, and $18.4 \%$ white (Caucasian). Overall, the proportion of the African/black population is at least $80 \%$ in all provinces except Western Cape and Northern Cape Provinces. The study protocol was approved by the University of Venda Research Ethics Committee (Study number SMNS/09/MBY/003). Signed informed consent was obtained from all participants before questionnaire administration for demographic data and blood collection. The experiments were undertaken with the understanding and written consent of each subject, and that 
the study conforms to the Code of Ethics of the World Medical Association (Declaration of Helsinki).

Two groups of patients were used for this study. The first group comprised 112 patients enrolled in an HIV diversity study for whom CD4 and viral load measurements were not available. These patients were not on treatment. The second group comprised 94 patients who were enrolled in a prospective HIV drug resistance study. Baseline analysis was done when patients were treatment naïve. Their treatment regimen comprised two nucleoside reverse transcriptase inhibitors, lamivudine, stavudine (d4T) and a non-nucleoside reverse transcriptase inhibitor, efavirenz. Viral genetic analysis, CD4 and viral load measurements were evaluated every three months for a period of 12 months in this group (Toga Laboratories, Johannesburg). The total number of patients available for pharmacogenetic analysis was 206.

\subsection{Genomic DNA amplification and genotyping of MDR1 C3435T and CYP2B6 G516T}

DNA was extracted from $5 \mathrm{ml}$ of blood in EDTA from each subject using the DNA mini kit (Qiagen) according to the manufacturer's instructions. A 363bp product encompassing the MDR1 gene was amplified using the following primers MDR1F 5'-GGCAGTTTC AGTGTAAGAAATAATG-3' (forward) and MDR1R 5'-TCAAACTATAGGCCAGAGAGGC-3' (reverse). Primers CYP2B6F 5'-AGGTGACAGCCTGATGTTC C-3' (forward) and CYP2B6R 5'-TTTCTCGTGTGTT CTGGGTG-3' (reverse) were used to amplify the CYP2B6 gene. The reaction mixture for both gene regions comprised a final concentration of $4 \mu \mathrm{M}$ of forward and reverse primers, $200 \mu \mathrm{M}$ dNTP's (Roche), $4 \mathrm{U}$ FastStart Taq polymerase (Roche), $10 \mathrm{X} \mathrm{Buffer}\left(\mathrm{MgCl}_{2}\right.$, $25 \mathrm{mM}$ ) (Roche), $5 \mu \mathrm{l}$ of DNA template and nuclease free water (Qiagen) in a $50 \mu \mathrm{l}$ total reaction volume. Cycling conditions were the same for both amplifications and comprised an initial denaturation at $94^{\circ} \mathrm{C}$ for $3 \mathrm{~min}$, followed by 35 cycles of denaturation at $94^{\circ} \mathrm{C}$ for $2 \mathrm{~min}$, annealing at $55^{\circ} \mathrm{C}$ and extension at $72^{\circ} \mathrm{C}$ for $2 \mathrm{~min}$; and final extension at $72^{\circ} \mathrm{C}$ for $10 \mathrm{~min}$. The resulting PCR products were visualized by UV transillumination on a $1 \%$ agarose gel stained with ethidium bromide.

\subsection{Genotyping}

The genotypes of MDR1 sequences were determined by comparing exon 26 with a wild type sequence [25], and a variant sequence $\mathrm{C} 3435 \mathrm{~T}$ (accession number NM_00097) obtained from the GenBank. Prior to this, purified PCR products were sequenced on both strands on a BiGDye Terminator Cycle Sequencer. Sequences were manually edited with SeqMan Pro Version 7.1 (DNASTAR). One hundred and ninety seven reliable sequences (197/206) were used in the genotypic analysis.

The CYP2B6 gene was genotyped by restriction fragment length polymorphism (RFLP) to detect substitution at position G516. DNA was digested with BSeNI (Fermentas) [26] according to the manufacturer's instructions. Genotypes were identified from the restriction digestion profiles and were classified as homozygous wild (GG), heterozygous (GT) and homozygote of variant (TT). One hundred and ninety nine DNA products were available for RFLP analysis. For confirmation of RFLP results, fifteen PCR products representing each of the genotypes observed were selected and sequenced.

\subsection{Statistical analysis}

Analysis of variance (ANOVA) and Tukey's test were used for multiple comparisons; Maulchy's sphericity test was used for covariance determination. Allele frequencies, expected genotypes and equilibrium of alleles in the population were analyzed by the HardyWeinberg method. A significance level of 0.05 was considered statistically significant.

\section{Results}

\subsection{Demographics of study population}

Of the 206 chronically infected HIV patients, 146 were females and 60 were males. The mean age was 36.8 years (range 18-59). The mean CD4 count was 152 per microlitre (range 1-700), and mean viral load was 128,638 copies per millilitre (range 1727->500,000). All the study subjects were black Africans.

\subsection{Frequency and distribution of MDR1 C3435 genotypes}

The expected product size of approximately $363 \mathrm{bp}$ comprising the complete MDR1 exon 26 gene was obtained for all 206 samples. Of the 206 DNA fragments, reliable sequences were obtained for 197 (95.6\%). In comparison with the wild type, 11 different SNP were 
Table 1

Observed and expected genotype frequencies of MDR1 C3435T and CYP2B6 G516T in South African subjects determined using the Hardy-Weinberg Method.

\begin{tabular}{|c|c|c|c|c|c|c|}
\hline Genotype & $\begin{array}{c}\text { Observed number of } \\
\text { genotypes (Frequency) }\end{array}$ & $\begin{array}{c}\text { Expected number of } \\
\text { genotypes }\end{array}$ & $\begin{array}{l}\text { Wild type allele } \\
\text { frequency }\end{array}$ & $\begin{array}{c}\text { Variant allele } \\
\text { frequency }\end{array}$ & $\mathrm{X}^{2}$ & p-value \\
\hline \multicolumn{2}{|c|}{ MDR1 C3435T } & & 0.89 & 0.11 & 0.974 & 0.324 \\
\hline $\mathrm{CC}$ & $155(0.787)$ & 156.3 & & & & \\
\hline $\mathrm{CT}$ & $41(0.208)$ & 38.3 & & & & \\
\hline $\mathrm{TT}$ & $01(0.005)$ & 2.3 & & & & \\
\hline \multicolumn{2}{|c|}{ CYP2B6 G516T } & & 0.51 & 0.49 & 65.204 & 0.000 \\
\hline GG & $24(0.12)$ & 52.5 & & & & \\
\hline GT & $156(0.785)$ & 99.9 & & & & \\
\hline TT & $19(0.095)$ & 47.5 & & & & \\
\hline
\end{tabular}

Where $p<0.05$ the expected and observed data are not consistent with HWE. HWE is not applicable if there are less than five events in the genotypic analysis. The frequencies of the genotypes in South African HIV infected subjects were calculated using the Hardy-Weinberg Method. For MDR1, the observed CC genotype (wild type) is more prevalent than the CT or TT variants and this is significant. The expected frequencies for each genotype are comparable to the observed values. For CYP2B6, the observed GT variant is the most prevalent when compared to GG or TT and this is significant. The expected frequencies for GG and TT are more than the observed and this was significant.
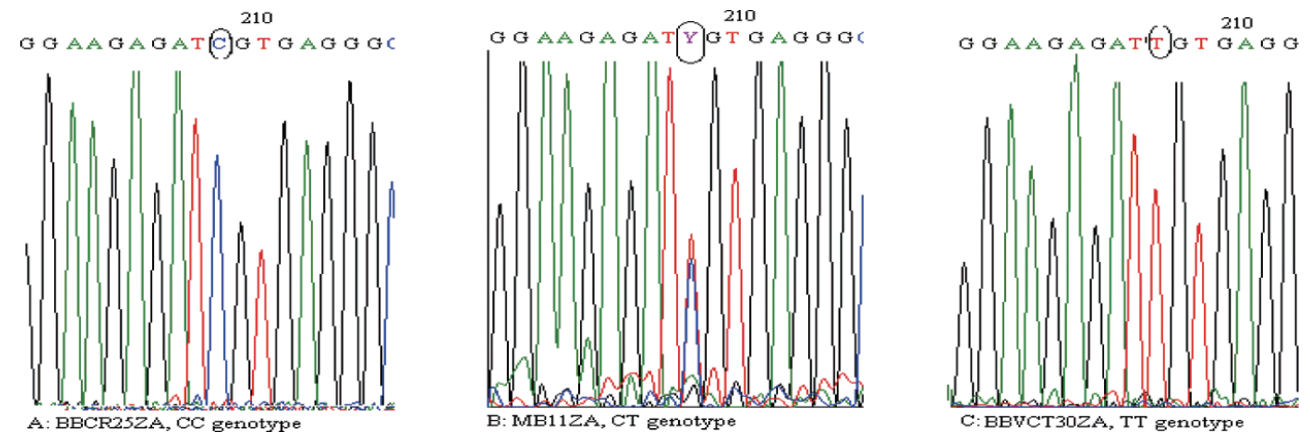

Fig. 1. Electropherograms of MDR1 exon 26 gene. Figure shows nucleotide substitutions at position C3435 of MDR1 exon 26 from black South African HIV infected subjects. A: Wild Type CC, B: Heterozygous genotype CT; C: Variant TT genotype. Circled bases indicate positions where base substitution occurs (B and C compared to A).

observed along exon 26 (data not shown). Genotypes and their relative frequencies included $\mathrm{CC}$ homozygous (78.7\%, 155/197); CT heterozygous (20.8\%; 41/197) and TT homozygous $(0.5 \%, 1 / 197)$. Although only one individual was homozygous for the mutant allele (TT), the Hardy-Weinberg calculation gave an expected frequency of 2.3. Figure 1 is a representation of three sequences with substitutions at position C3435. The distribution of observed and expected frequencies of MDR1 C3435 genotypes is presented in Table 1. The observed and expected genotype and allele frequencies did not show statistically significant differences compared to those expected under the Hardy-Weinberg Equilibrium (HWE) $\left(p=0.32\right.$ and $\left.X^{2}=0.974\right)$.

\subsection{Frequency and distribution of CYP2B6 G516 genotypes}

Restriction fragment length polymorphism analysis of the CYP2B6 G516 gene revealed four different re- striction patterns: One fragment of $289 \mathrm{bp}$ for the uncut product (TT) and two fragments of 196 and $93 \mathrm{bp}$ for the wild type (GG) and three or four fragments for the heterozygous product (GT). Twelve percent (24/199) of the study population had the homozygous GG genotype, 78.4\% (156/199) had the heterozygote GT genotype and $9.5 \%$ (19/199) had the homozygote TT genotype. The observed allele frequencies did not show statistically significant differences compared to those expected under HWE, whereas the observed genotype frequencies showed statistically significant differences when compared to the expected outcomes $(p=0.00$ with 1 degree of freedom, and $\left.\mathrm{X}^{2}=65.204\right)$. If $p<$ 0.05 , it is not consistent with HWE (See Table 1).

Sequence analysis for restriction sites were $100 \%$, $77.8 \%$, and $100 \%$ in agreement with the RFLP profiles of the GG (wild type), GT (heterozygote) and TT (variant) genotypes respectively (data not shown). Discordant RFLP GT genotypes were depicted as homozy- 


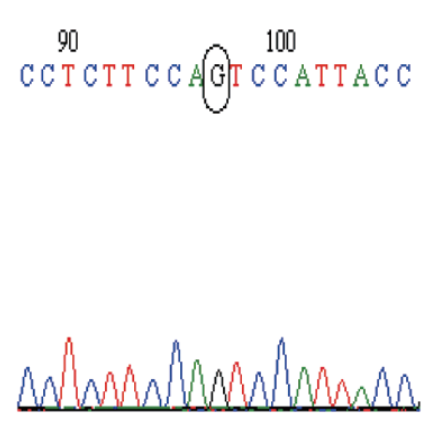

A: MB79, GG genotype

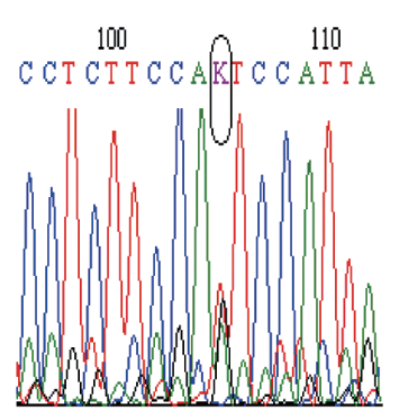

B:MB60, GT genotype
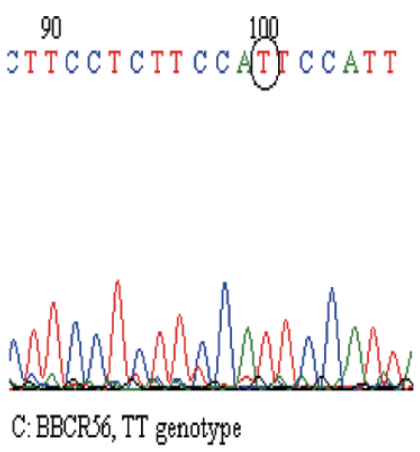

Fig. 2. Electropherograms of CYP2B6 exon 4 gene. Figure shows nucleotide sequences of CYP2B6 exon 4 at position G516 from black South African HIV infected subjects. A: Wild Type, GG genotype; B: Heterozygous GT genotype; C: Variant, TT Genotype. Circled bases indicate positions where base substitution occurs (B and C compared to A).

gotes of the mutant allele by sequencing (populationbased dideoxy method) (Fig. 2).

\subsection{Association of CD4 and viral load measurements with MDR1 C3435 and CYP2B6 G516 polymorphisms}

Overall, the 94 cohort patients were evaluated at baseline, and at 3, 6, 9 and 12 months post therapy initiation for immune recovery and viral load suppression respectively. The influence of polymorphisms on CD4 T cell count and plasma viral load measurements were done for 53 patients for whom at least a baseline and two follow up measurements at 3 month intervals were available. Fewer patients presented for the third and fourth follow-up visits and hence CD4 T cell count and viral load measurements for these time points were excluded from the statistical analyses. Therefore, multivariate analysis and repeated measures ANOVA testing was done for 53 patients. Multivariate analysis indicated the effect of time on increase in CD4 T-cell count $(F=62,55 ; p<0.0001)$. Therapy significantly reduced plasma viral load to undetectable levels $(<50$ cells $/ \mathrm{mL})$ in $75.5 \%(40 / 53)$ of the patients. The other patients had viral loads in the range of 52-25809 cells/mL after 9 months of therapy $(F=10.50, p<$ 0.0002). Repeated measures ANOVA indicate sufficient evidence of differences in means of CD4 T-cell count $(F=79.21 ; p<0.0001)$ and viral load measurements over time $(p<0.0001)$. There was no significant difference in increase in CD4 T-cell count and decrease in plasma viral load among patients irrespective of MDR1 C3435 (CC, CT and TT) and CYP2B6 G516 (GG, GT and TT) genotypes (data not shown). Factors such as gender, age and weight were not associated with increase in CD4 T cell count or plasma viral load decrease over time.

\subsection{Sequence accession numbers}

The sequence data reported here are available from GenBank under the following accession numbers: CYP2B6 exon 4: [GenBank: HQ291559-HQ291568]; MDR1 exon 26: [GenBank: HQ291569-HQ291765].

\section{Discussion and conclusion}

Human immunodeficiency virus (HIV) infected individuals require life-long treatment for continuous HIV1 viral suppression and immune restoration [27], and the continuous use of anti-HIV therapy has resulted in a significant decline in disease-related morbidity and mortality. However, virologic, immunologic, pharmacologic and pharmacokinetic differences between patients contribute to inter-patient variability in response to drugs. Single nucleotide polymorphisms responsible for key proteins involved in ARV drug metabolism (CYP450 enzymes), and transport (P-glycoprotein) are known to play a role in influencing therapy efficacy [28]. The TT genotype of CYP 2B6 is associated with reduced enzyme activity, whereas the TT genotype of MDR1 is correlated with the functionality of the protein.

Characterization of these genes and utilization of pharmacogenetic testing to identify different alleles may be an important tool for therapy optimization. This study investigated the frequency, distribution, and association of MDR1 C3435 and CYP2B6 G516 polymorphisms with immunological and virological responses in a group of HIV-1 infected South African patients.

The current investigation, with subjects from northern South Africa, observed a relatively high frequency (89\%) of MDR1 C3435 and a relatively low (11\%) 
frequency of MDR1 3435T alleles in the population studied. Similarly, in a study conducted in KwaZulu Natal, South Africa, the $\mathrm{C}$ allele was reported in $85 \%$ of African subjects as opposed to $41 \%$ in Indians (Asian origin), and 35\% of Caucasians [18]. The high prevalence of the $\mathrm{C}$ allele in the African subjects is in agreement with the findings of other investigators [13, $19,29,30]$, where frequencies of above $70 \%$ were described, as compared to about $30 \%$ in European and Asian subjects [30]. The inference is that the $\mathrm{C}$ allele is prevalent in black Africans irrespective of locality, thus emphasizing ethnic relatedness. The $\mathrm{C}$ allele is associated with increased $\mathrm{P}$-gp expression and thus reduced drug availability in cells infected by HIV, whereas the $\mathrm{T}$ allele is associated with better immune recovery after therapy initiation due to higher antiviral activity of the drugs $[4,19,31]$. Only one patient carried the $\mathrm{T}$ allele in the present study.

When comparing responses of patients with the CC or CT genotype, there was no genotype-dependent response visible because most patients responded well to therapy irrespective of a particular genotype (data not shown). However, viral load remained unsuppressed in some patients 24 weeks post therapy. More so, the observed lack of suppression was not genotype dependent. Since only one patient had the TT genotype, it was not possible to compare immune recovery of patients with the $\mathrm{CC}$ and $\mathrm{CT}$ genotypes.

The frequencies of CYP2B6 516G and T alleles were $51 \%$ and $49 \%$ with a GG genotype (extensive metaboliser) prevalence of $12 \%$, GT (intermediate metaboliser) genotype of $78.7 \%$ and $9.5 \%$ of TT (poor metaboliser) genotype. The frequency of the $\mathrm{T}$ allele is comparable to the $42 \%$ previously reported in West African subjects, as opposed to lower frequencies observed in Koreans (14\%), Caucasians (25\%), Japanese (16\%) and white Americans (28\%) [32]. The TT genotype is associated with decreased CYP2B6 expression and activity as well as low rate of EFV 8-hydroxylation. Studies confirming relatively higher persisting levels of EFV in Africans than in Whites found more CNS side effects one week after EFV initiation in people with TT genotype, which is more common among Africans [6, 12]. Patients with the TT genotype express low levels of EFV concentrations in the liver. Determination of genotypes before treatment with EFV-based therapy will assist in the prediction of plasma EFV concentration when prescribing EFV-based therapy [33].

Better immune recovery was observed in the present study population in individuals with GG, GT and TT of CYP2B6 position 516. This was evident due to increase in CD4 T cell counts of patients over time, after therapy initiation. However, the rate of increase was slightly lower in patients with GG and GT. Majority of the study participants had viral loads suppressed to undetectable levels irrespective of the genotype, and there was no significant difference in viral load decrease among these patients. Patients with the TT genotype are classified as slow metabolisers [13] and therefore we suspect that the low viral load counts observed might be due to persistently slow drug metabolism by CYP2B6. Although other CYPs such as CYP3A4 and CYP3A5 are implicated in the secondary pathway metabolism of EFV and NVP, metabolism of EFV is more exclusively through the CYP2B6 pathway [34-36]. Our findings did not show any difference in rate of decrease in viral load between patients with the TT and GG genotypes. Although the study did not investigate phenotypic outcomes, this observation may mean that these patients are slow metabolisers due to the observed decrease in viral loads. On the other hand, if information on adherence to therapy in terms of actual drug intake were obtained this could have provided additional data to explain the lack of relationship between TT or GG with a decrease in HIV RNA and CD4 count increase.

Although patients with CYP2B6 TT genotype had viral load suppressed to undetectable levels, this proportion of patients may experience therapeutic failure unless efavirenz dosage is adjusted. A limitation of this study is the lack of pharmacokinetic data. This could have enabled the measurement of plasma levels of the drugs and then correlate therapeutic outcomes of patients with a particular genotype. The second limitation is the small number of patients size $(n=53)$ available for association analysis; as a result a robust analysis of the association between genotypes and therapy outcome could not be ascertain, Hence, there is need for confirmation of the lack of association of CYP2B6 polymorphism with efavirenz and nevirapine in a larger study. However, this is the first report on the frequency and potential association of MDR1 C3435T and CYP2B6 G516T with therapy outcome in an African population of Northern South Africa, with the observation that MDR1 CC and CYP2B6 TT genotypes are prevalent in the studied population; and may not be associated with efavirenz and nevirapine treatment outcome.

\section{Authors' contributions}

TMM carried out DNA isolation, RFLP experiments, analyzed the sequences and wrote the manuscript, JN 
did DNA isolation and analyzed the sequences, DM and RNN wrote the manuscript, POB conceived the study, analyzed data and wrote the manuscript. All authors approved the manuscript. The authors have no competing interests.

\section{Acknowledgements}

We thank the National Department of Health and National Research Foundation of South Africa for financial support. We also thank the patients who participated in the study, the opinions expressed here are those of the authors.

\section{References}

[1] G. Severino amd M.D. Zompo, Adverse drug reactions, Role of Pharmacogenomics, Pharmacological Research 49 (2004), 363-373.

[2] V. Tozzi, Pharmacogenetics of antiretrovirals, Antiviral Research 85 (2010), 190-200.

[3] R.L. Oostendorp, J.H. Beijen and J.H.M. Schellens, The biological and clinical role of drug transporter at the intestinal barrier, Cancer Treatment Review 35 (2009), 137-147.

[4] E. Schaeffeler, E. Eichelbaum, M. Brinkmann, U. Penger, A. Asante-Poku, M.U. Zanger and M. Schwab, Frequency of C3435T polymorphism of MDR1 gene in African people, Lancet 358 (2001), 383-384.

[5] Department of Health, The South African antiretroviral treatment guidelines, Republic of South Africa (2010), 1-6.

[6] J. King and J.A. Aberg, Clinical impact of patient population differences and genomic variation in efavirenz therapy, AIDS 22 (2008), 1709-1717.

[7] M. Ingelman-Sundberg, S.C. Sim, A. Gomez and C. Rodriguez-Antona, Influence of cytochrome $\mathrm{P} 450$ polymorphisms on drug therapies, pharmacogenetic, pharmacoepigenetic and clinical aspects, Pharmacology and Therapeutics 116 (2007), 496-452.

[8] A. Saitoh, E. Sarles, E. Capparelli, F. Aweeka, A. Kovacs, S.K. Burchett, A. Wiznia, S. Nachman, T. Fenton and S.A. Spector, CYP2B6 genetic variants are associated with nevirapine and clinical response in HIV-1 infected children, AIDS 27 (2007), 2191-2199.

[9] D. Haas, L.M. Smeaton, R.W. Shafer, G.K. Robbins, G.D. Morse, L. Labbe, G.R. Wilkinson, D.B. Clifford, R.T. D’Aquila, V. De Gruttola, R.B. Pollard, T.C. Merigan, M.S. Hirsch, A.L. George, J.P. Donahue, R.B. Kim, Pharmacogenetics of long-term responses to antiretrovirals containing Efavirenz and/or Nelfinavir: an adult AIDS clinical trials group study, Journal of Infectious Diseases 192 (2005), 19311942.

[10] S. Rodriguez-Novoa, P. Barreiro, A. Rendon, I. JimenezNacher, J. Gonzalez-Lahoz and V. Soriano, Influence of $516 \mathrm{G}>\mathrm{T}$ polymorphisms at the gene encoding the CYP4502B6 isoenzyme on efavirenz plasma concentrations in HIVinfected subjects, Clinical Infectious Diseases 40 (2005), 1358-1361.
[11] S.T. Sadiq, S. Fredericks, S.H. Khoo, P. Rice and D.W. Holt, Efavirenz detectable in plasma 8 weeks after stopping therapy and subsequent development of non-nucleoside reverse transcriptase inhibitor-associated resistance, AIDS 19 (2005), 1716-1717.

[12] H.J. Ribaudo, D.W. Haas, C. Tierney, R.B. Kim, G.R. Wikinson, R. Gulick, D.B. Clifford, C. Marzolini, C.V. Fletcher, K.T. Tashima and D.R. Kuritzkes, Pharmacogenetics of plasma efavirenz exposure after treatment discontinuation: An adult AIDS clinical trials group study, Clinical Infectious Diseases 42 (2006), 401-407.

[13] C. Nyakutira, D. Roshammar, E. Chigutsa, P. Chonzi, M. Ashton, C. Nhachi and C. Masimirembwa, High prevalence of the CYP2B6 516 $\rightarrow \mathrm{T}(* 6)$ variant and effect on the population pharmacokinetics of efavirenz in HIV/AIDS outpatients in Zimbabwe, European Journal of Clinical Pharmacology 64 (2008), 357-365.

[14] S.R. Penzak, P. Mugyenyi, F. Mbamanya, V. Natarajan, R.M. Alfaro, C. Kityo, E. Formentin and E. Masur, Cytochrome P450 2B6 (CYP2B6) G516T influences nevirapine plasma concentrations in HIV-infected patients in Uganda, HIV Medicine 8 (2007), 86-91.

[15] J. Whetham, S. Charalambous, J. Lewis, K. Fielding, H. McIlleron, A. Walley, G.J. Churchyard and A.D. Grant, Efavirenz dose reduction based on CYP2B6 genotype is not supported by data from men in South Africa, HIV Medicine 9 (2008), 21.

[16] A, Owen, C. Goldring, P. Morgan, D. Chadwick, B.K. Park and M. Pirmohamed, Relationship between the C3435T and G2677T(A) polymorphisms in the ABCB1 gene and Pglycoprotein expression in human liver, British Journal of Clinical Pharmacology 59 (2005), 365-370.

[17] C.G.L. Lee and M.M. Gottseman, HIV-1 Protease inhibitors and the MDR1 drug transporter, Journal of Clinical Investigation 101 (1998), 287-288.

[18] P. Chelule, M. Gordon and T. Palanee, MDR1 and CYP3A4 polymorphisms among African, Indian, and white populations in KwaZulu-Natal, South Africa, Clinical Pharmacology and Therapeutics 74 (2003), 195-196.

[19] Q. Dong, B. Xu, Y. Tan, Z. Liu, L. Tian, B. Zhang, C. Lin, H. Kung, J.J.Y. Sung and M. He, The genetic variability of MDR1 C3435T polymorphisms in four Southern Chinese populations, Biomedicine and Pharmacotherapy 63 (2009), 658662.

[20] M.L. Bernal, B. Sinues, A. Fanlo and E. Mayayo, Frequency Distribution of C3435T Mutation in Exon 26 of the MDR1 Gene in a Spanish Population, Therapeutic Drug Monitoring 25 (2003), 107-111.

[21] M, Pirmohamed and J. Back, The pharmacogenomics of HIV, Pharmacogenomics Journal 1 (2001), 243-253.

[22] D. Haas, Pharmacogenomics and HIV Therapeutics, Journal of Infectious Diseases 191 (2005), 1397-1400.

[23] Y. Li, Y. Wang, Y. Li and L. Yang, MDR1 gene polymorphisms and clinical relevance, Acta Genetica Sinica 33 (2006), 93104.

[24] C. Balram, A. Sharma, C. Sivathasan and E.J.D. Lee, Frequency of C3435T single nucleotide MDR1 genetic polymorphism in an Asian population: Phenotypic-genotypic correlates, Journal of Clinical Pharmacology 56 (2003), 78-83.

[25] C. Chen, D. Clark, K. Ueda, I. Pastan, M.M. Gottesman and I.B. Roninson, Genomic organization of the Human multidrug resistance gene and origin of P-glycoproteins, Journal of Biological Chemistry 265 (1990), 506-514. 
[26] N. Ariyoshi, M. Miyazaki, K. Toide, Y. Sawamura and T. Kamataki, A single nucleotide polymorphism of CYP2B6 found in Japanese enhances catalytic activity by autoactivation, Biochemical and Biophysical Research Communications 281 (2001), 1256-1260.

[27] T.R. Cressey and M. Lallemant, Pharmacogenetics of antiretroviral drugs for the treatment of HIV-infected patients: An update, Infections, Genetics and Evolution 7 (2007), 333342.

[28] K.E. Estes, K.H. Busse and S.R. Penzak, Pharmacogenetic considerations in the management of HIV infection, Journal of Pharmacy Practice 20 (2007), 234-245.

[29] K.L. Fung and M.M. Gottesman, A synonymous polymorphism in a common MDR (ABCB) haplotype shapes protein function, Biochimica Biophysica Acta 1794 (2009), 860-871.

[30] M.M. Ameyaw, F. Regateiro, X. Liu, M. Tariq, A. Mobarek, N. Thornton, G.O. Folayan, J. Githang'a, A. Indalo, D. OforiAdjei, D.A. Price-Evans and H.L. McLeod, MDR1 pharmacogenetics, frequency of the C3435T mutation in exon 26 is significantly influenced by ethnicity, Pharmacogenetics 11 (2001), 217-221

[31] J. Fellay, C. Marzolini, E.R. Meaden, D.J. Back, T. Buclin, J. Chave, L.A. Decosterd, H. Furrer, M. Opravil, G. Pantaleo, D. Retelska, L. Ruiz, A.H. Schinkel, P. Vernazza and C.B. Eap, A. Telenti for the Swiss HIV cohort study, Response to antiretroviral treatment in HIV-1 Infected individuals with allelic variants of the multidrug resistance transporter 1: a pharmacogenetic study, Lancet 359 (2002), 31-36.

[32] G. Ramachandran, K. Ramesh, A.K.H. Kumar, P. Kumar,
K. Ramesh, S. Anitha, G. Narendran, P. Menon, C. Gomathi and S. Swaminathan, Association of high frequency of CYP2B6 G516T polymorphism among ethnic South Indian HIV-infected patients with elevated plasma efavirenz and nevirapine, Journal of Antimicrobial Chemotherapy 63 (2009), 841-843.

[33] K. Tsuchiya, H. Gatanaga, N. Tachikawa, K. Teruya, Y. Kikuchi, M. Yoshino, T. Kuwahara, T. Shirasaka, S. Kimura and S. Oka, Homozygous CYP2B6 *6 (Q172H and K262R) correlates with high plasma efavirenz concentrations in HIV-1 patients treated with standard efavirenz-containing regimens, Biochemical and Biophysical Research Communications 319 (2004), 1322-1326.

[34] B.A. Ward, J.C. Gorski, D.R. Jones, S.D. Hall, D.A. Flockhart and Z. Desta, The cytochrome P450 2B6 (CYP2B6) is the main catalyst of efavirenz primary and secondary metabolism: implication for HIV/AIDS therapy and utility of efavirenz as a substrate marker of CYP2B6 catalytic activity, Journal of Pharmacology and Experimental Therapeutics 306 (2003), 287-300.

[35] P.F Smith, R. DiCenzo and G.D. Morse, Clinical pharmacokinetics of non-nucleoside reverse transcriptase inhibitors, Clinical Pharmacokinetics 40 (2001), 893-905.

[36] S. Uttayamakul, S. Likanonsakul, W. Manosuthi, N. Wichukchinda, T. Kalambaheti, E.E. Nakayama, T. Shioda and S. Khusmith, Effects of CYP2B6 G516T polymorphisms on plasma efavirenz and nevirapine levels when co-administered with rifampicin in HIV/TB co-infected Thai adults, AIDS Research and Therapy 7 (2010), 8. 


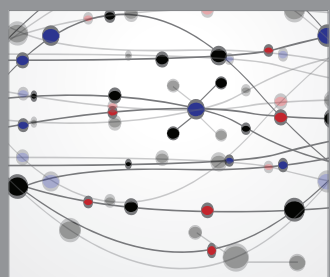

The Scientific World Journal
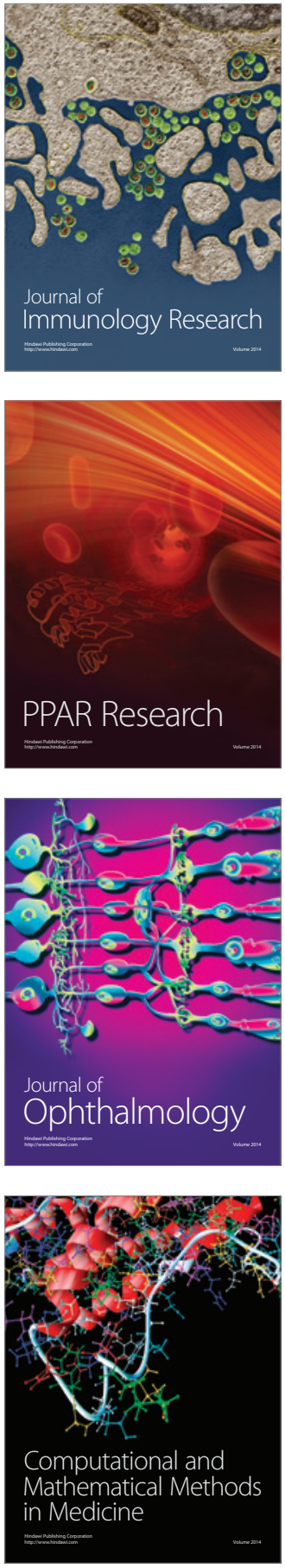

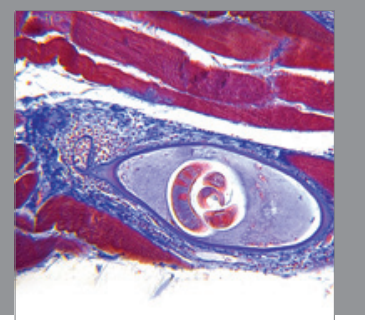

Gastroenterology

Research and Practice
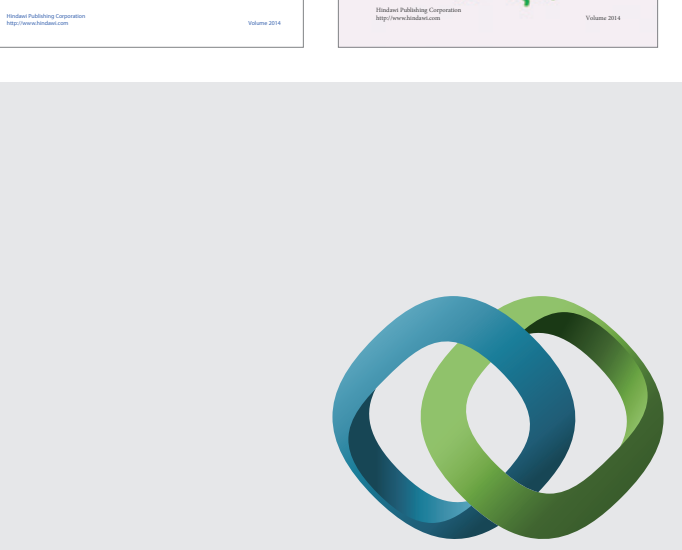

\section{Hindawi}

Submit your manuscripts at

http://www.hindawi.com
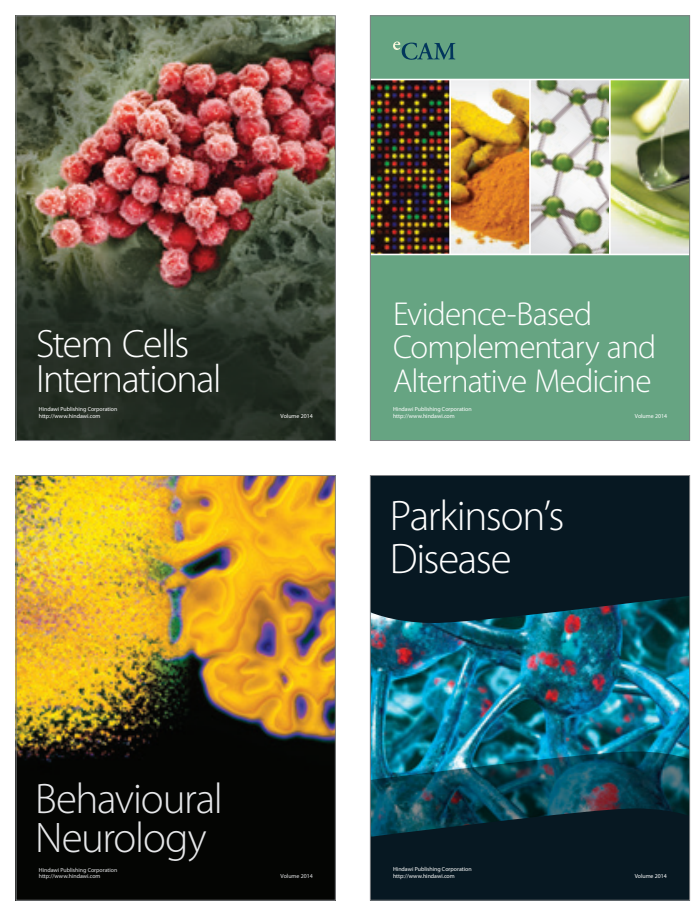

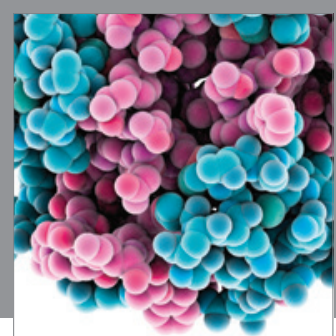

Journal of
Diabetes Research

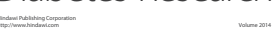

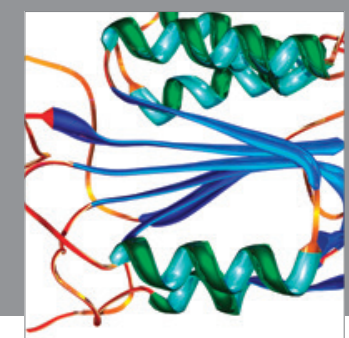

Disease Markers
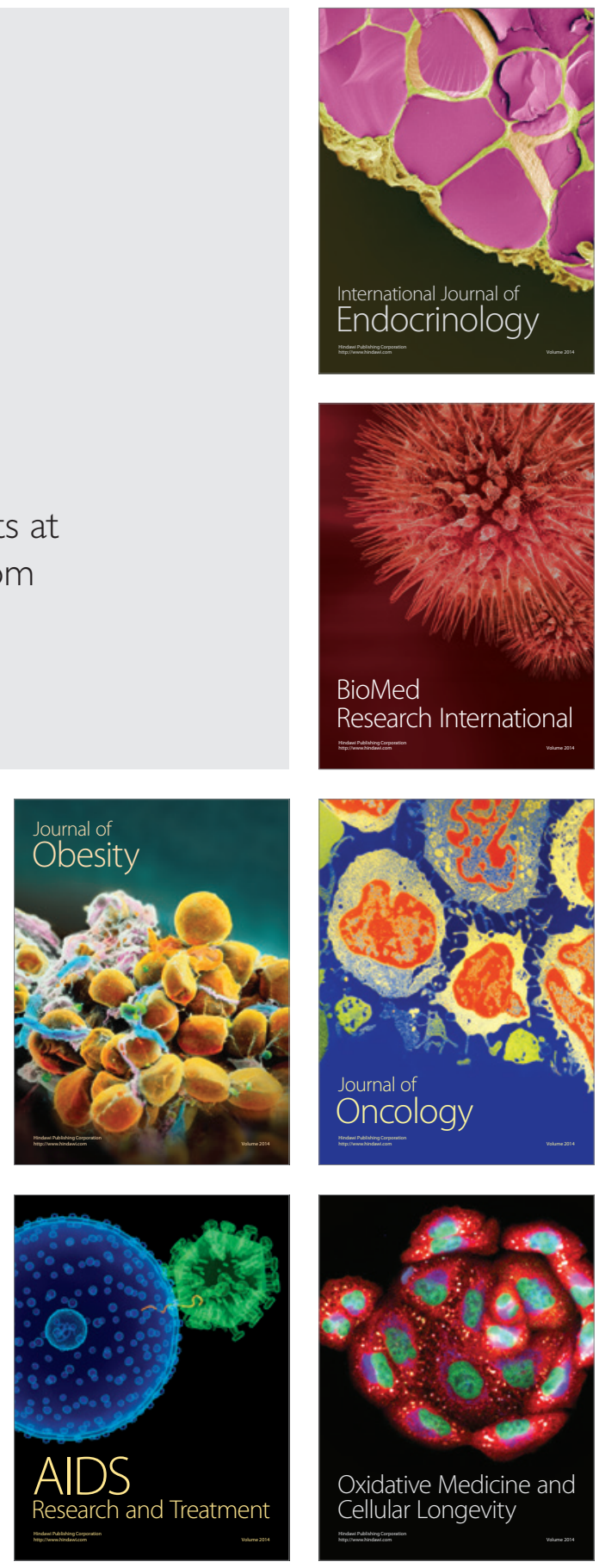